ANNALES

UNIVERSITATIS MARIAE CURIE-SKŁODOWSKA

L UBLIN - POLONIA

VOL. LXV, NO. 1, 2011

SECTIO A

$11-19$

ANNA BEDNARSKA

\title{
Lagrangians and Euler morphisms on fibered-fibered frame bundles from projectable-projectable classical linear connections
}

\begin{abstract}
We classify all $\mathcal{F}^{2} \mathcal{M}_{m_{1}, m_{2}, n_{1}, n_{2}}$-natural operators $A$ transforming projectable-projectable torsion-free classical linear connections $\nabla$ on fibered-fibered manifolds $Y$ of dimension $\left(m_{1}, m_{2}, n_{1}, n_{2}\right)$ into $r$ th order Lagrangians $A(\nabla)$ on the fibered-fibered linear frame bundle $L^{\text {fib-fib }}(Y)$ on $Y$. Moreover, we classify all $\mathcal{F}^{2} \mathcal{M}_{m_{1}, m_{2}, n_{1}, n_{2}}$-natural operators $B$ transforming projectable-projectable torsion-free classical linear connections $\nabla$ on fiberedfibered manifolds $Y$ of dimension $\left(m_{1}, m_{2}, n_{1}, n_{2}\right)$ into Euler morphism $B(\nabla)$ on $L^{\text {fib-fib }}(Y)$. These classifications can be expanded on the $k$ th order fiberedfibered frame bundle $L^{\text {fib-fib,k}}(Y)$ instead of $L^{\text {fib-fib }}(Y)$.
\end{abstract}

1. Introduction. Lagrangians and Euler morphisms are important tools in the variational calculus. Several physical theories are using Euler-Lagrange equations, which are related with the Euler morphism of an $r$ th order Lagrangian on a fibered manifold.

The idea of Lagrangians and Euler morphisms in the case of fibered manifolds was described in [2]. The aim of the present note is the generalization of results which were reached in [1] to the case of fibered-fibered manifolds.

2000 Mathematics Subject Classification. 58A20, 53A55, 53C05.

Key words and phrases. Fibered-fibered manifold, Lagrangian, Euler morphism, natural operator, classical linear connection. 
2. Fibered-fibered manifolds. A fibered-fibered manifold $Y$ is any commutative diagram

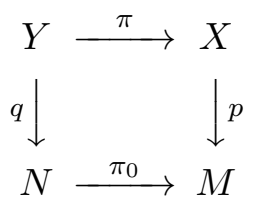

where maps $\pi, \pi_{0}, q, p$ are surjective submersions and induced map $Y \rightarrow$ $X \times_{M} N, y \mapsto(\pi(y), q(y))$ is a surjective submersion. A fibered-fibered manifold has dimension $\left(m_{1}, m_{2}, n_{1}, n_{2}\right)$ if $\operatorname{dim} Y=m_{1}+m_{2}+n_{1}+n_{2}$, $\operatorname{dim} X=m_{1}+m_{2}, \operatorname{dim} N=m_{1}+n_{1}, \operatorname{dim} M=m_{1}$. For two fiberedfibered manifolds $Y_{1}, Y_{2}$ of the same dimension $\left(m_{1}, m_{2}, n_{1}, n_{2}\right)$, a morphism $f: Y_{1} \rightarrow Y_{2}$ is quadruple of local diffeomorphisms $f: Y_{1} \rightarrow Y_{2}, f_{1}: X_{1} \rightarrow X_{2}$, $f_{2}: N_{1} \rightarrow N_{2}, f_{0}: M_{1} \rightarrow M_{2}$ such that all squares of the cube in question are commutative [3].

All fibered-fibered manifolds of the given dimension $\left(m_{1}, m_{2}, n_{1}, n_{2}\right)$ and their all morphisms form the category which we denote by $\mathcal{F}^{2} \mathcal{M}_{m_{1}, m_{2}, n_{1}, n_{2}}$.

Every object from the category $\mathcal{F}^{2} \mathcal{M}_{m_{1}, m_{2}, n_{1}, n_{2}}$ is locally isomorphic to the standard fibered-fibered manifold

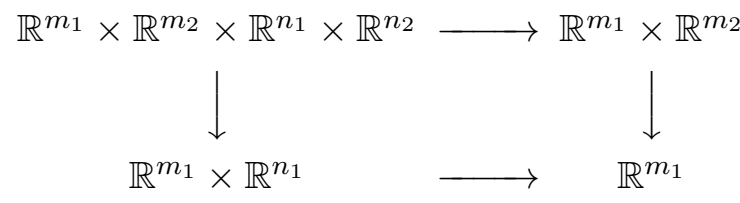

which we denote by $\mathbb{R}^{m_{1}, m_{2}, n_{1}, n_{2}}$, where arrows are obvious projections.

For fibered-fibered manifold $Y$ we have the fibered-fibered linear frame bundle

$L^{\text {fib-fib }}(Y)=\left\{j_{(0,0,0,0)}^{1} \psi \mid \psi: \mathbb{R}^{m_{1}, m_{2}, n_{1}, n_{2}} \rightarrow Y\right.$ is an $\mathcal{F}^{2} \mathcal{M}_{m_{1}, m_{2}, n_{1}, n_{2}}$-map $\}$

with the jet target projection $\pi_{Y}: L^{\mathrm{fib}-\mathrm{fib}}(Y) \rightarrow Y$,

$$
\pi_{Y}\left(j_{(0,0,0,0)}^{1} \psi\right)=\psi(0,0,0,0),
$$

where $(0,0,0,0) \in \mathbb{R}^{m_{1}+m_{2}+n_{1}+n_{2}}$. The bundle $L^{\mathrm{fib}-\mathrm{fib}}(Y)$ is a principal bundle over $Y$ with a structure group $G_{m_{1}, m_{2}, n_{1}, n_{2}}^{1}=L_{(0,0,0,0)}^{\mathrm{fib}-\mathrm{fib}}\left(\mathbb{R}^{m_{1}, m_{2}, n_{1}, n_{2}}\right)$ acting on the right on $L^{\text {fib-fib }}(Y)$ by the composition of jets. Every $\mathcal{F}^{2} \mathcal{M}_{m_{1}, m_{2}, n_{1}, n_{2}}$-map $f: Y \rightarrow Y_{1}$ induces a fibered map (a principal bundle morphism) $L^{\text {fib-fib }}(f): L^{\text {fib-fib }}(Y) \rightarrow L^{\text {fib-fib }}\left(Y_{1}\right)$ over $f$ by the composition of jets $L^{\mathrm{fib}-\mathrm{fib}}(f)\left(j_{(0,0,0,0)}^{1} \psi\right)=j_{(0,0,0,0)}^{1}(f \circ \psi)$. The correspondence

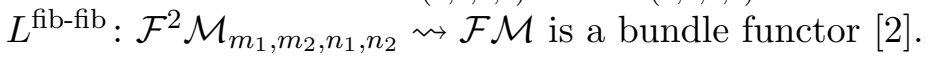

\section{Lagrangians and natural operators transforming connections} into Lagrangians. An $r$ th order Lagrangian on a fibered manifold $p$ : 
$X \rightarrow M$ is a base preserving morphism $\lambda: J^{r}(X) \rightarrow \wedge^{m} T^{*} M$ from the $r$-jet prolongation bundle

$$
J^{r}(X)=\left\{j_{x}^{r} \sigma \mid \sigma: M \rightarrow X \text { is a local section of } p: X \rightarrow M, x \in M\right\}
$$

into the bundle $\wedge^{m} T^{*} M$ of $m=\operatorname{dim} M$-forms on $M$ [2].

A classical linear connection $\widetilde{\nabla}$ on a fibered manifold $p: X \rightarrow M$ is projectable if there exists a (unique) classical linear connection $\widetilde{\nabla}$ on $M$ such that a connection $\widetilde{\nabla}$ is $p$-related with a connection $\widetilde{\nabla}$, that is $T p \circ\left(\widetilde{\nabla}_{W} Z\right)=$ $\left(\underline{\widetilde{\nabla}}_{W} \underline{Z}\right) \circ p$, where $W$ and $Z$ are projectable vector fields on $X$, which are $p$-related with vector fields $\underline{W}$ and $\underline{Z}$ on $M$.

Let $Y$ be a fibered-fibered manifold

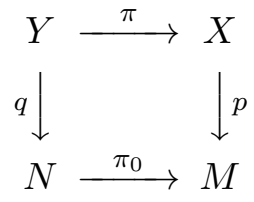

We say that a projectable classical linear connection $\nabla$ on $Y$ is projectableprojectable if there exists a unique projectable classical linear connection $\underline{\nabla}$ on $X$ such that a connection $\nabla$ is $\pi$-related with a connection $\nabla$.

In this paper we study the problem how a projectable-projectable torsion-free classical linear connection $\nabla$ on a fibered-fibered manifold $Y$ of dimension $\left(m_{1}, m_{2}, n_{1}, n_{2}\right)$ can induce an $r$ th order Lagrangian $A(\nabla)$ on $\pi_{Y}: L^{\mathrm{fib}-\mathrm{fib}}(Y) \rightarrow Y$ in the canonical way. To this aim we must determine $\mathcal{F}^{2} \mathcal{M}_{m_{1}, m_{2}, n_{1}, n_{2}}$-natural operators

$$
A: Q_{\tau}^{\text {proj-proj }} \rightarrow\left(J^{r} L^{\text {fib-fib }}, \wedge^{m} T^{*}\right),
$$

where $m=m_{1}+m_{2}+n_{1}+n_{2}$, in the sense of [2].

We describe completely all such natural operators $A$ in question.

An $\mathcal{F}^{2} \mathcal{M}_{m_{1}, m_{2}, n_{1}, n_{2}}$-natural operator

$$
A: Q_{\tau}^{\text {proj-proj }} \rightarrow\left(J^{r} L^{\text {fib-fib }}, \wedge^{m} T^{*}\right)
$$

(where $m=m_{1}+m_{2}+n_{1}+n_{2}$ ) sending projectable-projectable torsion-free classical linear connections $\nabla$ on fibered-fibered manifolds $Y$ of dimension $\left(m_{1}, m_{2}, n_{1}, n_{2}\right)$ into $r$ th order Lagrangians $A(\nabla)$ on the fibered-fibered linear frame bundle $\pi_{Y}: L^{\mathrm{fib}-\mathrm{fib}}(Y) \rightarrow Y$ for $Y$ is the family of $\mathcal{F}^{2} \mathcal{M}_{m_{1}, m_{2}, n_{1}, n_{2}}{ }^{-}$ invariant regular operators

$$
A_{Y}: Q_{\tau}^{\text {proj-proj }}(Y) \rightarrow \operatorname{Lagr}^{r}\left(L^{\mathrm{fib}-\mathrm{fib}}(Y)\right)
$$

for $\mathcal{F}^{2} \mathcal{M}_{m_{1}, m_{2}, n_{1}, n_{2}}$-objects $Y$, where $Q_{\tau}^{\text {proj-proj }}(Y)$ is the space of all projectable-projectable torsion-free classical linear connections on $Y$ and $\operatorname{Lagr}^{r}\left(L^{\mathrm{fib}-\mathrm{fib}}(Y)\right)$ is the space of all $r$ th order Lagrangians on

$$
\pi_{Y}: L^{\mathrm{fib}-\mathrm{fib}}(Y) \rightarrow Y .
$$


The invariance means that if $\nabla \in Q_{\tau}^{\text {proj-proj }}(Y)$ and $\nabla_{1} \in Q_{\tau}^{\text {proj-proj }}\left(Y_{1}\right)$ are $f$-related with respect to an $\mathcal{F}^{2} \mathcal{M}_{m_{1}, m_{2}, n_{1}, n_{2}}$-map $f: Y \rightarrow Y_{1}$, then $A_{Y}(\nabla)$ and $A_{Y_{1}}\left(\nabla_{1}\right)$ are also $f$-related. The regularity of a natural operator $A_{Y}$ means that $A_{Y}$ transforms smoothly parametrized families of connections in $Q_{\tau}^{\text {proj-proj }}(Y)$ into smoothly parametrized families of Lagrangians in $\operatorname{Lagr}^{r}\left(L^{\mathrm{fib}-\mathrm{fib}}(Y)\right)$.

To present an example of natural operator $A$ in question we need some preparations. Let $M$ be an $m$-manifold with a torsion-free classical linear connection $\tilde{\widetilde{\nabla}}$. Given a linear frame $\underline{l} \in L(M)$, the linear isomorphism $\underline{l}: \mathbb{R}^{m} \rightarrow T_{\underline{x}}(M)$ defines a coordinate system in $T_{\underline{x}}(M)$. Therefore, $\underline{\widetilde{\nabla}}-$ exponential map $\operatorname{Exp} \underline{\underline{\nabla}}: T_{\underline{x}}(M) \rightarrow M$ being the diffeomorphism, defines a normal coordinate system $\underline{\varphi}$ with center $\underline{x}$ in $M$ by the composition $\underline{\varphi}=$ $\underline{l}^{-1} \circ(\operatorname{Exp} \underline{\underline{\bar{x}}})^{-1}: M \rightarrow \mathbb{R}^{m}$. If $\underline{\varphi}_{1}: M \rightarrow \mathbb{R}^{m}$ is another $\underline{\tilde{\nabla}}$-normal coordinate system on $M$ with center $\underline{x}$, then $\underline{\varphi}_{1}=I \circ \underline{\varphi}$ for some linear isomorphism $I: \mathbb{R}^{m} \rightarrow \mathbb{R}^{m}[4]$.

Let $p: X \rightarrow M$ be a fibered manifold of dimension $(m, n)$ and let $x \in X_{\underline{x}}$, $\underline{x} \in M$. Let $\widetilde{\nabla}$ be a projectable torsion-free classical linear connection on $X$ with the underlying torsion-free classical linear connection $\underline{\widetilde{\nabla}}$ on $M$. Since a connection $\widetilde{\nabla}$ is $p$-related with $\underline{\widetilde{\nabla}}$, then $p$ sends $\widetilde{\nabla}$-geodesics into $\underline{\widetilde{\nabla}}$-geodesics. Consequently, the $\widetilde{\nabla}$-exponential map $\operatorname{Exp}_{x} \widetilde{\nabla}: T_{x}(X) \rightarrow X$ at $x$ is a local fibered diffeomorphism covering the $\widetilde{\nabla}$-exponential map $\operatorname{Exp} \underline{\underline{\bar{v}}}: T_{\underline{x}}(M) \rightarrow M$ at $\underline{x}$, where $T_{x}(X)$ is treated as a fibered manifold $T p: \bar{T}_{x}(\bar{X}) \rightarrow T_{\underline{x}}(M)$.

If we compose $\left(\operatorname{Exp}_{x} \tilde{\nabla}\right)^{-1}$ with a fibered linear isomorphism (fibered linear frame) $l: \mathbb{R}^{m, n} \rightarrow T_{x}(X)$ covering a linear frame $\underline{l}: \mathbb{R}^{m} \rightarrow T_{\underline{x}}(M)$, then we obtain a fibered $\widetilde{\nabla}$-normal coordinate system $\varphi=l^{-1} \circ\left(\operatorname{Exp}_{x}^{\widetilde{\nabla}}\right)^{-1}: X \rightarrow$ $\mathbb{R}^{m, n}$ with center $x$ covering a $\underline{\widetilde{\nabla}}$-normal coordinate system

$$
\underline{\varphi}=\underline{l}^{-1} \circ\left(\operatorname{Exp}_{\underline{\underline{x}}}\right)^{-1}: M \rightarrow \mathbb{R}^{m}
$$

with center $\underline{x}$. If $\varphi_{1}: X \rightarrow \mathbb{R}^{m, n}$ is another fibered $\widetilde{\nabla}$-normal coordinate system on $X$ with center $x$, then $\varphi_{1}=I \circ \varphi$ for some fibered linear isomorphism $I: \mathbb{R}^{m, n} \rightarrow \mathbb{R}^{m, n}$.

Quite similarly as above, if $\nabla$ is a projectable-projectable torsion-free classical linear connection on a fibered-fibered manifold $Y$ of dimension $\left(m_{1}, m_{2}, n_{1}, n_{2}\right)$ and $y \in Y$, then there exists a fibered-fibered $\nabla$-normal coordinate system $\varphi: Y \rightarrow \mathbb{R}^{m_{1}, m_{2}, n_{1}, n_{2}}$ with center $y$. If $\varphi_{1}: Y \rightarrow \mathbb{R}^{m_{1}, m_{2}, n_{1}, n_{2}}$ is another fibered-fibered $\nabla$-normal coordinate system with center $y$, then $\varphi_{1}=I \circ \varphi$ for some fibered-fibered linear isomorphism $I: \mathbb{R}^{m_{1}, m_{2}, n_{1}, n_{2}} \rightarrow$ $\mathbb{R}^{m_{1}, m_{2}, n_{1}, n_{2}}$. 
4. The first main result. Let $Q_{\text {proj-proj }}^{s}$ be the vector space of all $s$ jets $j_{(0,0,0,0)}^{s}(\nabla)$ at $(0,0,0,0) \in \mathbb{R}^{m_{1}+m_{2}+n_{1}+n_{2}}$ of projectable-projectable torsion-free classical linear connections $\nabla$ on $\mathbb{R}^{m_{1}, m_{2}, n_{1}, n_{2}}$ satisfying the condition

$$
\sum_{j, k=1}^{m_{1}+m_{2}+n_{1}+n_{2}} \nabla_{j k}^{i}(x) x^{j} x^{k}=0 \quad \text { for } \quad i=1, \ldots, m_{1}+m_{2}+n_{1}+n_{2},
$$

where $\nabla_{j k}^{i}: \mathbb{R}^{m_{1}, m_{2}, n_{1}, n_{2}} \rightarrow \mathbb{R}$, for $i, j, k=1, \ldots, m_{1}+m_{2}+n_{1}+n_{2}$, are the Christoffel symbols of a connection $\nabla$ in the usual fibered-fibered coordinate system $x^{1}, \ldots, x^{m_{1}+m_{2}+n_{1}+n_{2}}$ on $\mathbb{R}^{m_{1}, m_{2}, n_{1}, n_{2}}$.

Equivalently, $x^{1}, \ldots, x^{m_{1}+m_{2}+n_{1}+n_{2}}$ are fibered-fibered $\nabla$-normal coordinates with center $(0,0,0,0)$. The equivalence is a simple consequence of the well-known equations of $\nabla$-geodesics and the fact that in the $\nabla$-normal coordinate system $\nabla$-geodesics passing through the center are straight lines.

Let $\pi_{s}: Q_{\text {proj-proj }}^{\infty} \rightarrow Q_{\text {proj-proj }}^{s}$ for $s=1,2, \ldots$ be the jet projections. Let $\pi_{0}^{r}: J^{r}\left(L^{\mathrm{fib}-\mathrm{fib}}\left(\mathbb{R}^{m_{1}, m_{2}, n_{1}, n_{2}}\right)\right) \rightarrow L^{\mathrm{fib}-\mathrm{fib}}\left(\mathbb{R}^{m_{1}, m_{2}, n_{1}, n_{2}}\right)$ be the jet projection.

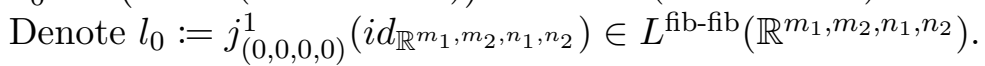

Let $J_{l_{0}}^{r}\left(L^{\mathrm{fib}-\mathrm{fib}}\left(\mathbb{R}^{m_{1}, m_{2}, n_{1}, n_{2}}\right)\right)$ be the fibre of $\pi_{0}^{r}$ at $l_{0}$. Let $\mu: Q_{\text {proj-proj }}^{\infty} \times$ $J_{l_{0}}^{r}\left(L^{\text {fib-fib }}\left(\mathbb{R}^{m_{1}, m_{2}, n_{1}, n_{2}}\right)\right) \rightarrow \mathbb{R}$. We say that $\mu$ satisfies the finite determination property, if for any $\rho \in Q_{\text {proj-proj }}^{\infty}$ and $\sigma \in J_{l_{0}}^{r}\left(L^{\mathrm{fib}-\mathrm{fib}}\left(\mathbb{R}^{m_{1}, m_{2}, n_{1}, n_{2}}\right)\right)$ we can find an open neighborhood $U \subset Q_{\text {proj-proj }}^{\infty}$ of $\rho$, open neighborhood $V \subset J_{l_{0}}^{r}\left(L^{\mathrm{fib}-\mathrm{fib}}\left(\mathbb{R}^{m_{1}, m_{2}, n_{1}, n_{2}}\right)\right)$ of $\sigma$, a finite number $s$ and a smooth map $f: \pi_{s}(U) \times V \rightarrow \mathbb{R}$ such that $\mu=f \circ\left(\pi_{s} \times i d_{V}\right)$ on $U \times V$.

We are in a position to present the following example of the operator $A$ in question.

Example 1. Let $\mu: Q_{\text {proj-proj }}^{\infty} \times J_{l_{0}}^{r}\left(L^{\mathrm{fib}-\mathrm{fib}}\left(\mathbb{R}^{m_{1}, m_{2}, n_{1}, n_{2}}\right)\right) \rightarrow \mathbb{R}$ be a function satisfying the finite determination property. Given a projectable-projectable torsion-free classical linear connection $\nabla$ on a fibered-fibered manifold $Y$ of dimension $\left(m_{1}, m_{2}, n_{1}, n_{2}\right)$, we define the $r$ th order Lagrangian

$$
A_{Y}^{\langle\mu\rangle}(\nabla): J^{r}\left(L^{\mathrm{fib}-\mathrm{fib}}(Y)\right) \rightarrow \wedge^{m} T^{*}(Y)
$$

on $\pi_{Y}: L^{\mathrm{fib}-\mathrm{fib}}(Y) \rightarrow Y$ by

$A_{Y}^{\langle\mu\rangle}(\nabla)(\sigma):=\mu\left(j_{(0,0,0,0)}^{\infty}\left(\varphi_{*} \nabla\right), J^{r}\left(L^{\mathrm{fib}-\mathrm{fib}}(\varphi)\right)(\sigma)\right) \cdot l_{1}^{*} \wedge \ldots \wedge l_{m_{1}+m_{2}+n_{1}+n_{2}}^{*}$,

where $m=m_{1}+m_{2}+n_{1}+n_{2}, \sigma \in J_{l}^{r}\left(L^{\mathrm{fib}-\mathrm{fib}}(Y)\right), l=j_{(0,0,0,0)}^{r}\left(\varphi^{-1}\right) \in$ $\left(L^{\mathrm{fib}-\mathrm{fib}}(Y)\right)_{y}, y \in Y, l_{i}=T\left(\varphi^{-1}\right)\left(\left.\frac{\partial}{\partial x^{i}}\right|_{(0,0,0,0)}\right)$ for $i=1, \ldots, m_{1}+m_{2}+$ $n_{1}+n_{2}$ is the basis in $T_{y}(Y)$ and $l_{i}^{*}$ for $i=1, \ldots, m_{1}+m_{2}+n_{1}+n_{2}$ is the dual basis in $T_{y}^{*}(Y)$ and $\varphi: Y \rightarrow \mathbb{R}^{m_{1}, m_{2}, n_{1}, n_{2}}$ is a fibered-fibered $\nabla$-normal coordinate system with center $y$ such that $J^{r}\left(L^{\text {fib-fib }}(\varphi)\right)(\sigma) \in$ $J_{l_{0}}^{r}\left(L^{\mathrm{fib}-\mathrm{fib}}\left(\mathbb{R}^{m_{1}, m_{2}, n_{1}, n_{2}}\right)\right)$. 
The definition of $A_{Y}^{\langle\mu\rangle}(\nabla)(\sigma)$ is correct because $\operatorname{germ}_{y}(\varphi)$ is uniquely determined.

Consequently, for given a projectable-projectable torsion-free classical linear connection $\nabla$ on $Y$, we have an $r$ th order Lagrangian

$$
A_{Y}^{\langle\mu\rangle}: J^{r}\left(L^{\mathrm{fib}-\mathrm{fib}}(Y)\right) \rightarrow \wedge^{m} T^{*}(Y), \quad \text { where } m=m_{1}+m_{2}+n_{1}+n_{2} .
$$

The family $A^{\langle\mu\rangle}: Q_{\tau}^{\text {proj-proj }} \rightsquigarrow\left(J^{r} L^{\text {fib-fib }}, \wedge^{m} T^{*}\right)$ of operators

$$
\begin{aligned}
A_{Y}^{\langle\mu\rangle}: Q_{\tau}^{\text {proj-proj }}(Y) & \longrightarrow \operatorname{Lagr}^{r}\left(L^{\text {fib-fib }}(Y)\right), \\
\nabla & \longrightarrow A_{Y}^{\langle\mu\rangle}(\nabla)
\end{aligned}
$$

for any $\mathcal{F}^{2} \mathcal{M}_{m_{1}, m_{2}, n_{1}, n_{2}}$-object $Y$ is an $\mathcal{F}^{2} \mathcal{M}_{m_{1}, m_{2}, n_{1}, n_{2}}$-natural operator.

The main result of the present note is the following:

Theorem 1. Any $\mathcal{F}^{2} \mathcal{M}_{m_{1}, m_{2}, n_{1}, n_{2}}$-natural operator

$$
A: Q_{\tau}^{\text {proj-proj }} \rightsquigarrow\left(J^{r} L^{\text {fib-fib }}, \wedge^{m} T^{*}\right),
$$

where $m=m_{1}+m_{2}+n_{1}+n_{2}$, is of the form $A=A^{\langle\mu\rangle}$ for a uniquely determined function $\mu: Q_{\text {proj-proj }}^{\infty} \times J_{l_{0}}^{r}\left(L^{\text {fib-fib }}\left(\mathbb{R}^{m_{1}, m_{2}, n_{1}, n_{2}}\right)\right) \rightarrow \mathbb{R}$ satisfying the finite determination property.

Proof. Let $A$ be a $\mathcal{F}^{2} \mathcal{M}_{m_{1}, m_{2}, n_{1}, n_{2}}$-natural operator in question. We must define a map $\mu: Q_{\text {proj-proj }}^{\infty} \times J_{l_{0}}^{r}\left(L^{\text {fib-fib }}\left(\mathbb{R}^{m_{1}, m_{2}, n_{1}, n_{2}}\right)\right) \rightarrow \mathbb{R}$ by

$$
\mu\left(j_{(0,0,0,0)}^{\infty}(\nabla), \sigma\right):=\left\langle A_{\mathbb{R}^{m_{1}, m_{2}, n_{1}, n_{2}}}(\nabla)(\sigma),\left(l_{0}\right)_{1} \wedge \ldots \wedge\left(l_{0}\right)_{m_{1}+m_{2}+n_{1}+n_{2}}\right\rangle,
$$

where $l_{0}=\left(\left(l_{0}\right)_{1}, \ldots,\left(l_{0}\right)_{m_{1}+m_{2}+n_{1}+n_{2}}\right)$ is the basis in $T_{(0,0,0,0)}\left(\mathbb{R}^{m_{1}+m_{2}+n_{1}+n_{2}}\right)$. Then by the non-linear Peetre theorem [2], $\mu$ satisfies the finite determination property. By the invariance of $A$ and $A^{\langle\mu\rangle}$ with respect to fiberedfibered normal coordinates we obtain $A=A^{\langle\mu\rangle}$.

Remark 1. Quite similarly one can describe all $\mathcal{F}^{2} \mathcal{M}_{m_{1}, m_{2}, n_{1}, n_{2}}$-natural operators $A: Q_{\tau}^{\text {proj-proj }} \rightarrow\left(J^{r} L^{\text {fib-fib, } k}, \wedge^{m} T^{*}\right)$ transforming projectable-projectable torsion-free classical linear connections $\nabla$ on $\mathcal{F}^{2} \mathcal{M}_{m_{1}, m_{2}, n_{1}, n_{2}}$-objects $Y$ into $r$ th order Lagrangians $A_{Y}(\nabla)$ on $\pi_{Y}^{k}: L^{\text {fib-fib, } k}(Y) \rightarrow Y$, where

$$
\begin{aligned}
& L^{\text {fib-fib }, k}(Y) \\
& :=\left\{j_{(0,0,0,0)}^{k}(\psi) \mid \psi: \mathbb{R}^{m_{1}, m_{2}, n_{1}, n_{2}} \rightarrow Y \text { is a local } \mathcal{F}^{2} \mathcal{M}_{m_{1}, m_{2}, n_{1}, n_{2}} \text {-map }\right\}
\end{aligned}
$$

is the fibered-fibered $k$ th order frame bundle for $Y$. All such natural operators in question are of the form $A^{\langle\mu\rangle}$ for functions

$$
\mu: Q_{\text {proj-proj }}^{\infty} \times J_{l_{0}}^{r}\left(L^{\mathrm{fib-fib,k}}\left(\mathbb{R}^{m_{1}, m_{2}, n_{1}, n_{2}}\right)\right) \rightarrow \mathbb{R}
$$


satisfying the obviously modified finite determination property, where $J_{l_{0}}^{r}\left(L^{\mathrm{fib}-\mathrm{fib}, k}\left(\mathbb{R}^{m_{1}, m_{2}, n_{1}, n_{2}}\right)\right)$ is the fiber of

$$
J_{l_{0}}^{r}\left(L^{\mathrm{fib}-\mathrm{fib}, k}\left(\mathbb{R}^{m_{1}, m_{2}, n_{1}, n_{2}}\right)\right) \rightarrow L^{\mathrm{fib}-\mathrm{fib}, k}\left(\mathbb{R}^{m_{1}, m_{2}, n_{1}, n_{2}}\right)
$$

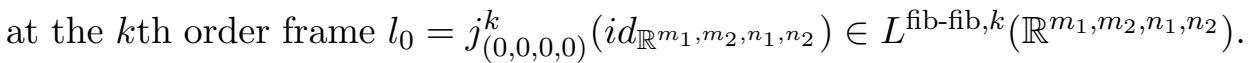
The generalized natural operators $A^{\langle\mu\rangle}$ can be defined quite similarly as in Example 1.

5. Euler morphisms and natural operators transforming connections into Euler morphisms. We recall that the $r$ th order Euler morphism on a fibered manifold $p: X \rightarrow M$ is a base preserving morphism $E: J^{r}(X) \rightarrow V^{*}(X) \otimes \wedge^{m} T^{*}(M)$, where $m=\operatorname{dim} M$. Here $V^{*}(X)$ denotes the vector bundle dual to the vertical vector bundle $V(X)$ for $X$. Special Euler morphisms can be obtained from Lagrangians by means of the well-known Euler operator [2], [5].

Quite similarly as for Lagrangians, we can describe completely all $\mathcal{F}^{2} \mathcal{M}_{m_{1}, m_{2}, n_{1}, n_{2}}$-natural operators

$$
B: Q_{\tau}^{\text {proj-proj }} \rightarrow\left(J^{r} L^{\text {fib-fib }}, V^{*} L^{\text {fib-fib }} \otimes \wedge^{m} T^{*}\right),
$$

where $m=m_{1}+m_{2}+n_{1}+n_{2}$, transforming projectable-projectable torsionfree classical linear connections $\nabla$ on fibered-fibered manifold $Y$ of dimension $\left(m_{1}, m_{2}, n_{1}, n_{2}\right)$ into $r$ th order Euler morphisms $B_{Y}(\nabla)$ on

$$
\pi_{Y}: L^{\mathrm{fib}-\mathrm{fib}}(Y) \rightarrow Y
$$

\section{The second main result.}

Example 2. We consider a function

$$
\mu: Q_{\text {proj-proj }}^{\infty} \times J_{l_{0}}^{r}\left(L^{\mathrm{fib}-\mathrm{fib}}\left(\mathbb{R}^{m_{1}, m_{2}, n_{1}, n_{2}}\right)\right) \rightarrow\left(\mathcal{L}\left(G_{m_{1}, m_{2}, n_{1}, n_{2}}^{1}\right)\right)^{*}
$$

satisfying the obviously modified finite determination property, where $\mathcal{L}\left(G_{m_{1}, m_{2}, n_{1}, n_{2}}^{1}\right)$ denotes the Lie algebra of Lie group $G_{m_{1}, m_{2}, n_{1}, n_{2}}^{1}$. Given a projectable-projectable torsion-free classical linear connection on a fiberedfibered manifold $Y$ of dimension $\left(m_{1}, m_{2}, n_{1}, n_{2}\right)$, we define an $r$ th order Euler morphism $B_{Y}^{\langle\mu\rangle}(\nabla): J^{r}\left(L^{\mathrm{fib}-\mathrm{fib}}(Y)\right) \rightarrow V^{*}\left(L^{\mathrm{fib}-\mathrm{fib}}(Y)\right) \otimes \wedge^{m} T^{*}(Y)$, where $m=m_{1}+m_{2}+n_{1}+n_{2}$, on $\pi_{Y}: L^{\mathrm{fib}-\mathrm{fib}}(Y) \rightarrow Y$ by

$$
\begin{aligned}
& \left\langle B_{Y}^{\langle\mu\rangle}(\nabla)(\sigma), \eta_{\mid l}^{*}\right\rangle \\
& \quad=\left\langle\mu\left(j_{(0,0,0,0)}^{\infty}\left(\varphi_{*} \nabla\right), J^{r}\left(L^{\mathrm{fib}-\mathrm{fib}}(\varphi)\right)(\sigma)\right), \eta\right\rangle l_{1}^{*} \wedge \ldots \wedge l_{m_{1}+m_{2}+n_{1}+n_{2}}^{*}
\end{aligned}
$$

for all $\sigma \in\left(J_{l}^{r}\left(L^{\mathrm{fib}-\mathrm{fib}}(Y)\right), l=\left(l_{1}, \ldots, l_{m}\right) \in\left(L^{\mathrm{fib}-\mathrm{fib}}(Y)\right)_{y}, y \in Y\right.$, where $m=m_{1}+m_{2}+n_{1}+n_{2}, \eta \in \mathcal{L}\left(G_{m_{1}, m_{2}, n_{1}, n_{2}}^{1}\right)$, where $\eta^{*}$ is the (vertical) fundamental vector field on the principal $G_{m_{1}, m_{2}, n_{1}, n_{2}}^{1}$-bundle $L^{\text {fib-fib }}(Y)$ corresponding to $\eta$ and $l_{1}^{*}, \ldots, l_{m}^{*} \in T_{y}^{*} Y$ is the dual basis to $l_{1}, \ldots, l_{m} \in T_{y} Y$ 
and $\varphi: Y \rightarrow \mathbb{R}^{m_{1} m_{2}, n_{1}, n_{2}}$ is a fibered-fibered $\nabla$-normal coordinate system on $Y$ with center $y$ such that $\varphi(y)=(0,0,0,0) \in \mathbb{R}^{m_{1}+m_{2}+n_{1}+n_{2}}$ and $J^{r}\left(L^{\mathrm{fib}-\mathrm{fib}}(\varphi)\right)(\sigma) \in\left(J_{l_{0}}^{r}\left(L^{\mathrm{fib}-\mathrm{fib}}\left(\mathbb{R}^{m_{1}, m_{2}, n_{1}, n_{2}}\right)\right)\right)$. The correspondence $B^{\langle\mu\rangle}: Q_{\tau}^{\text {proj-proj }} \rightsquigarrow\left(J^{r} L^{\text {fib-fib }}, V^{*} L^{\text {fib-fib }} \otimes \wedge^{m} T^{*}\right)$, where $m=m_{1}+m_{2}+$ $n_{1}+n_{2}$, is $\mathcal{F}^{2} \mathcal{M}_{m_{1}, m_{2}, n_{1}, n_{2}}$-natural operator.

Similarly as Theorem 1 one can prove the following:

Theorem 2. Any $\mathcal{F}^{2} \mathcal{M}_{m_{1}, m_{2}, n_{1}, n_{2}}$-natural operator

$$
B: Q_{\tau}^{\text {proj-proj }} \rightsquigarrow\left(J^{r} L^{\text {fib-fib }}, V^{*} L^{\text {fib-fib }} \otimes \wedge^{m} T^{*}\right),
$$

where $m=m_{1}+m_{2}+n_{1}+n_{2}$, is of the form $B=B^{\langle\mu\rangle}$ for some uniquely determined function

$$
\mu: Q_{\text {proj-proj }}^{\infty} \times J_{l_{0}}^{r}\left(L^{\mathrm{fib}-\mathrm{fib}}\left(\mathbb{R}^{m_{1}, m_{2}, n_{1}, n_{2}}\right)\right) \rightarrow\left(\mathcal{L}\left(G_{m_{1}, m_{2}, n_{1}, n_{2}}^{1}\right)\right)^{*}
$$

satisfying the modified finite determination property.

Proof. Similarly as in the proof of Theorem 1 we define

$$
\mu: Q_{\text {proj-proj }}^{\infty} \times J_{l_{0}}^{r}\left(L^{\mathrm{fib}-\mathrm{fib}}\left(\mathbb{R}^{m_{1}, m_{2}, n_{1}, n_{2}}\right)\right) \rightarrow\left(\mathcal{L}\left(G_{m_{1}, m_{2}, n_{1}, n_{2}}^{1}\right)\right)^{*}
$$

by

$$
\begin{aligned}
& \left\langle\mu\left(j_{(0,0,0,0)}^{\infty}(\nabla), \sigma\right), \eta\right\rangle
\end{aligned}
$$

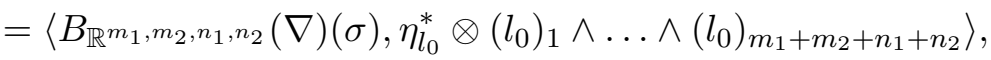

where

$$
\eta \in \mathcal{L}\left(G_{m_{1}, m_{2}, n_{1}, n_{2}}^{1}\right), j_{(0,0,0,0)}^{\infty}(\nabla) \in Q_{\text {proj-proj }}^{\infty}, \sigma \in J_{l_{0}}^{r}\left(L^{\mathrm{fib}-\mathrm{fib}}\left(\mathbb{R}^{m_{1}, m_{2}, n_{1}, n_{2}}\right)\right),
$$

$\eta^{*}$ is the fundamental vector field on $L^{\mathrm{fib}-\mathrm{fib}}\left(\mathbb{R}^{m_{1}, m_{2}, n_{1}, n_{2}}\right)$ corresponding to $\eta \in \mathcal{L}\left(G_{m_{1}, m_{2}, n_{1}, n_{2}}^{1}\right)$ and $l_{0}=\left(\left(l_{0}\right)_{1}, \ldots,\left(l_{0}\right)_{m_{1}+m_{2}+n_{1}+n_{2}}\right)$ is the basis in $T_{(0,0,0,0)}\left(\mathbb{R}^{m_{1}+m_{2}+n_{1}+n_{2}}\right)$. Then $B=B^{\langle\mu\rangle}$.

Remark 2. Quite similarly one can describe all $\mathcal{F}^{2} \mathcal{M}_{m_{1}, m_{2}, n_{1}, n_{2}}$-natural operators $B: Q_{\tau}^{\text {proj-proj }} \rightarrow\left(J^{r} L^{\text {fib-fib, } k}, V^{*} L^{\text {fib-fib, } k} \otimes \wedge^{m} T^{*}\right)$, where $m=$ $m_{1}+m_{2}+n_{1}+n_{2}$, transforming projectable-projectable torsion-free classical linear connections $\nabla$ on $\left(m_{1}, m_{2}, n_{1}, n_{2}\right)$-dimensional fibered-fibered manifolds $Y$ into Euler morphisms $B_{Y}(\nabla)$ on $\pi_{Y}^{k}: L^{\text {fib-fib, } k}(Y) \rightarrow Y$ of fiberedfibered frames of order $k$ of $Y$. All such natural operators are of the form $B^{\langle\mu\rangle}$ for all

$$
\mu: Q_{\text {proj-proj }}^{\infty} \times J_{l_{0}}^{r}\left(L^{\mathrm{fib}-\mathrm{fib}, k}\left(\mathbb{R}^{m_{1}, m_{2}, n_{1}, n_{2}}\right)\right) \rightarrow \mathcal{L}\left(G_{m_{1}, m_{2}, n_{1}, n_{2}}^{k}\right)
$$

satisfying the obviously modified finite determination property. The natural operators $B^{\langle\mu\rangle}$ can be constructed similarly as in Example 2. 


\section{REFERENCES}

[1] Kurek, J., Mikulski, W. M., Lagrangians and Euler morphisms from connections on the frame bundle, Proceedings of the XIX International Fall Workshop on Geometry and Physics, Porto, 2010.

[2] Kolář, I., Michor, P. W. and Slovák, J., Natural Operations in Differential Geometry, Springer-Verlag, Berlin, 1993.

[3] Kolář, I., Connections on fibered squares, Ann. Univ. Mariae Curie-Skłodowska Sect. A 59 (2005), 67-76.

[4] Kobayashi, S., Nomizu, K., Foundations of Differential Geometry, Vol. I, Interscience Publisher, New York-London, 1963.

[5] Kurek, J., Mikulski, W. M., On the formal Euler operator from the variational calculus in fibered-fibered manifolds, Proc. of the 6 International Conference Aplimat 2007, Bratislava, 223-229.

Anna Bednarska

Institute of Mathematics

Maria Curie-Skłodowska University

pl. Marii Curie-Skłodowskiej 1

20-031 Lublin

Poland

e-mail: bednarska@hektor.umcs.lublin.pl

Received May 5, 2010 